\title{
Beyond the Standard Model Higgs boson searches using the ATLAS detector
}

\author{
I.I. Tsukerman, on behalf the ATLAS Collaboration ${ }^{1, a}$ \\ ${ }^{1}$ Institute of Theoretical and Experimental Physics (ITEP), Moscow, 117218, Russia
}

\begin{abstract}
The discovery of a Higgs boson with a mass of about $125 \mathrm{GeV}$ has prompted the question of whether or not this particle is part of a larger and more complex Higgs sector than that envisioned in the Standard Model. In this talk, the current results from the ATLAS experiment on Beyond the Standard Model (BSM) Higgs boson searches are outlined. The results are interpreted in well-motivated BSM Higgs frameworks.
\end{abstract}

\section{Introduction}

A new boson with a mass of $125 \mathrm{GeV}$ was discovered by the ATLAS [1] and CMS [2] Collaborations at the Large Hadron Collider [3] (LHC) two years ago. All measured properties of this particle are compatible with the Standard Model (SM) predictions for the Higgs boson [4]. This discovery was a great success of the SM. However, the SM does not explain particle mass hierarchy, dark matter, dark energy, baryon asymmetry of the Universe and has problems with the unification of fundamental interactions [4]. Different extensions of the SM were proposed by theorists to solve these problems. Many of these extensions contain five Higgs bosons (neutral light and neutral heavy CP-even states, $h$ and $H$, one CP-odd neutral state $A$ and two charged states, $H^{+}$and $H^{-}$). Four masses $m_{h}, m_{H}$, $m_{A}, m_{H^{ \pm}}$, mixing angle $\alpha$ between the light and the heavy neutral Higgs boson, and the ratio $\tan \beta$ of two vacuum expectation values are the usual free parameters. ATLAS performs [5] both charged and neutral Higgs boson searches in different decay modes. This report contains a brief summary of these searches and is organized as follows. Section 2 is devoted to charged Higgs boson. The search modes include the decays $H^{+} \rightarrow \tau v, H^{+} \rightarrow c \bar{s}$ and $H^{+} \rightarrow h^{0} W$. In Section 3 neutral Higgs bosons are considered. Associated $Z H$-production is used to search for invisible decays of the $H$ boson. The decay channels which allowed to discover the SM-like Higgs boson $h^{0}, h \rightarrow \gamma \gamma, h \rightarrow Z Z^{\star} \rightarrow 4 \ell$, $h \rightarrow W W^{\star} \rightarrow \ell v \ell v$, are also used to search for neutral heavy CP-even Higgs boson, $H$. The $\tau \tau$ decay mode is used to detect both CP-even and CP-odd states, $h^{0}, H^{0}$ and $A^{0}$. Finally, the results of $X \rightarrow h h \rightarrow b \bar{b} \gamma \gamma$ searches are presented in Section 4 .

\section{Charged Higgs boson searches}

In the Minimal Super Symmetric Model (MSSM) a relation between top quark mass $m_{\text {top }}$ and $m_{H^{ \pm}}$ dictates both the production mode and decay channels of $H^{ \pm}$. If $m_{H^{ \pm}} \leq m_{t o p}$, the charged Higgs boson

ae-mail: Ilya.Tsukerman@ cern.ch

This is an Open Access article distributed under the terms of the Creative Commons Attribution License 4.0, which permits unrestricted use, distribution, and reproduction in any medium, provided the original work is properly cited. 
appears in the decay $t \rightarrow b H$, and the final state is $H b W b$. For $\tan \beta<1(\tan \beta>1)$, the decay $H^{+} \rightarrow c \bar{s}$ $\left(H^{+} \rightarrow \tau v\right)$ dominates. If $m_{H^{ \pm}} \geq m_{t o p}$, the $H^{+}$is produced together with $b$ - and $t$-quarks in the gluon fusion process. The decay $H^{+} \rightarrow \tau v$ is expected to have a large branching ratio.

\subsection{Search for $H^{+} \rightarrow c \bar{s}$ decays in $t \bar{t}$ events at $7 \mathrm{TeV}$ [6]}

Here one looks for events with a pair production of top quarks $(t \bar{t})$, in which one top quark goes into $b W$, with subsequent decay $W \rightarrow \ell v^{1}$, and the other decays into $b H^{+}$. The search mass range is 90 $150 \mathrm{GeV}$. The signal signature includes one isolated high- $p_{T}$ lepton, two energetic jets with invariant mass $m_{j j}=m_{H}$ and large missing transverse energy $E_{T}^{m i s s}$. Backgrounds consist of top-quark pair production $(t \bar{t})$, single top production, pair production of vector bosons, $Z+$ jets production and multi-jet production. Fig. 1a shows the di-jet invariant mass distribution. No deviations from the SM are seen. The 95\% CL exclusion limit on $B R(t \rightarrow H b)$ lies between 0.01 and 0.05 , depending on $m_{H^{+}}$, see Fig. 1b.

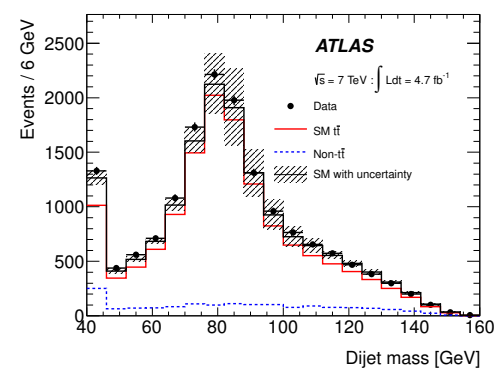

(a)

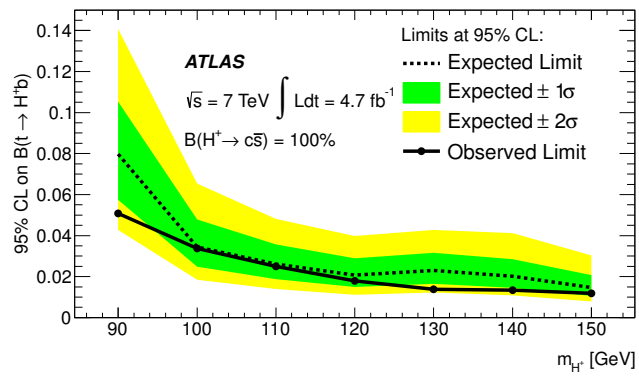

(b)

Figure 1. Results of the $H^{+} \rightarrow c \bar{s}$ search in $t \bar{t}$ events at $7 \mathrm{TeV}$ [6]. (a) The di-jet invariant mass distribution. (b) The $95 \%$ CL exclusion limit on the branching ratio of the $t \rightarrow H b$ decay as a function of $m_{H^{+}}$.

\subsection{Search for $H^{+} \rightarrow \tau v$ in $t \bar{t}$ events at $8 \mathrm{TeV}$ [7]}

The search mass ranges for $H^{+} \rightarrow \tau v$ decay in $t \bar{t}$ events are 90-160 GeV (low mass range) and 180$600 \mathrm{GeV}$ (high mass range). The signal signatures for the low and high mass ranges are four and three high- $p_{T}$ jets, respectively, together with a high- $p_{T} \tau$-lepton with hadronic decay and a large $E_{T}^{\text {miss }}$. Backgrounds include $t \bar{t}_{-}, W t$-, single top-, $W+$ jets-, $Z / \gamma^{\star}+$ jets-, di-boson- and multijet production. The exclusion limits under assumption of $B R\left(H^{+} \rightarrow \tau v\right)=1$ are presented in Fig. 2 and 3. Fig. 2 shows the $95 \% \mathrm{CL}$ limits on $B R\left(t \rightarrow b H^{+}\right)\left(H^{+}\right.$production cross section, $\left.\sigma_{H^{+}}\right)$as a function of $m_{H^{+}}$ for the low (high) mass cases. Depending on the $m_{H^{+}}$, the limit on the $B R\left(t \rightarrow b H^{+}\right)$is between $0.24 \%$ and $2.1 \%$ and the limit on $\sigma_{H^{+}}$is between $0.017 \mathrm{pb}$ and $0.9 \mathrm{pb}$. The limits on $\tan \beta$ as a function of $m_{H^{+}}$in the specific MSSM $m_{h}^{\max }$ scenario [8] are shown separately for the low and high mass cases in Fig. 3. The region above $\tan \beta=1$ is excluded for the charged Higgs boson with a mass 100-140 $\mathrm{GeV}$. In the high mass case, the exclusion region is $\tan \beta>47-63$ for $200 \mathrm{GeV} \leq m_{H^{ \pm}} \leq 300 \mathrm{GeV}$.

\footnotetext{
${ }^{1} \ell$ stands for charged lepton throughout the note.
} 


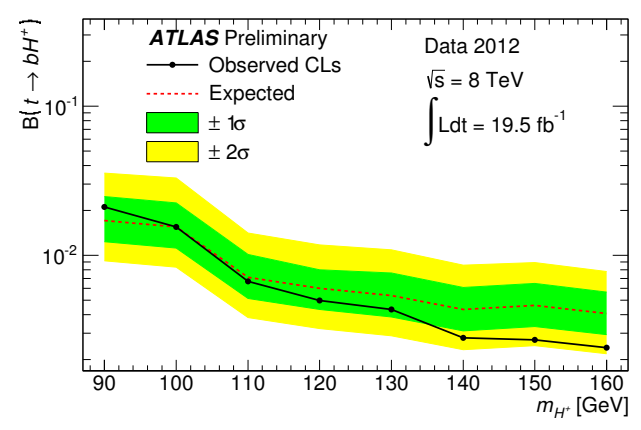

(a)

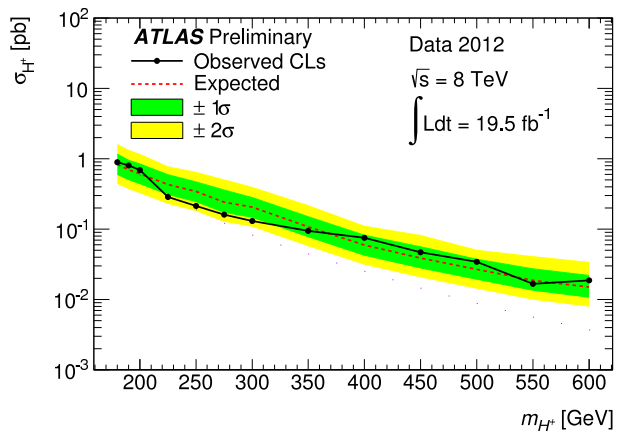

(b)

Figure 2. Exclusion limits for charged Higgs boson under the assumption $B R(H \rightarrow \tau v)=1$ [7]. (a) The 95\% CL exclusion limit on the branching ratio of the $t \rightarrow H b$ decay as a function of $m_{H^{+}}$in the range 90-160 GeV. (b) The $95 \%$ CL exclusion limit on the $\sigma_{H}$ as a function of $m_{H^{+}}$in the range 180-600 GeV.

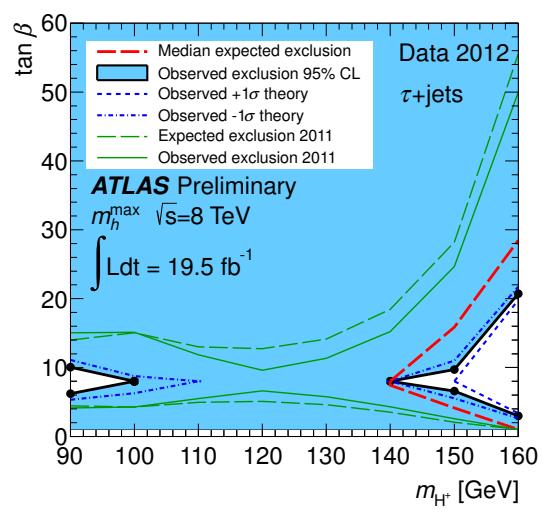

(a)

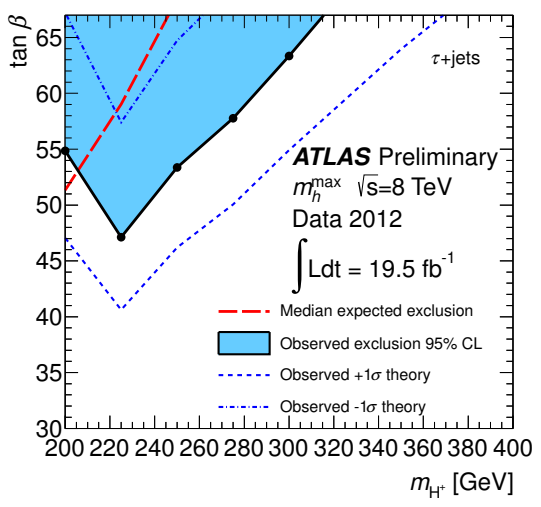

(b)

Figure 3. Exclusion limits for charged Higgs boson in the MSSM $m_{h}^{\max }$ scenario under the assumption $B R(H \rightarrow$ $\tau v)=1$ [7]. (a) The $95 \%$ CL exclusion limit on $\tan \beta$ as a function of $m_{H^{+}}$in the range $90-160 \mathrm{GeV}$. (b) The $95 \%$ CL exclusion limit on $\tan \beta$ as a function of $m_{H^{+}}$in the range $200-400 \mathrm{GeV}$.

\subsection{Search for cascade decay $H^{0} \rightarrow W H^{ \pm} \rightarrow h^{0} W W \rightarrow b \bar{b} W W$ at $8 \mathrm{TeV}$ [9]}

The search mass range for the cascade decay $H^{0} \rightarrow W H^{ \pm} \rightarrow h^{0} W W \rightarrow b \bar{b} W W$ (Fig. 4a) is 225$925 \mathrm{GeV}(325 \mathrm{GeV}-1025 \mathrm{GeV})$ for the $H^{ \pm}\left(H^{0}\right)$. The signature is one isolated high- $p_{T}$ lepton (from the $W$ decay), four high- $p_{T}$ jets, two of them being $b$-jets with invariant mass equal to $m_{h^{0}}$, a large $E_{T}^{m i s s}$ and the transverse mass $m_{T}^{W}$ depending on a lepton flavour ${ }^{2}$. The dominant background is from

\footnotetext{
${ }^{2}$ The $m_{T}^{W}$ is defined as $m_{T}^{W}=2 p_{T}^{\ell} p_{T}^{v}\left(1-\cos \left(\phi^{\ell}-\phi^{v}\right)\right.$ ), where $p_{T}^{\ell}\left(p_{T}^{v}\right)$ is lepton (neutrino) transverse momentum, $\phi^{\ell}\left(\phi^{v}\right)$ is lepton (neutrino) azimuthal angle. It is assumed that $p_{T}^{v}=E_{T}^{\text {miss }}$.
} 
$t \bar{t}$-production, with smaller contributions from the $W+$ jets-, $Z / \gamma^{\star}+$ jets-, di-boson-, single topand multijet production. A multivariate analysis based on seven kinematic variables is performed to discriminate the Higgs boson signal from the $t \bar{t}$-background. No deviations from the SM are seen. This transforms into the exclusion plot in Fig. $4 \mathrm{~b}$ which shows the $95 \%$ CL upper limit on the $\sigma_{H^{0}}$ multiplied by the related branching ratio as a function of $H^{ \pm}$and $H^{0}$ masses. The limit lies in the region between $0.065 \mathrm{pb}$ and $43 \mathrm{pb}$ under the assumption $m_{h^{0}}=125 \mathrm{GeV}$.

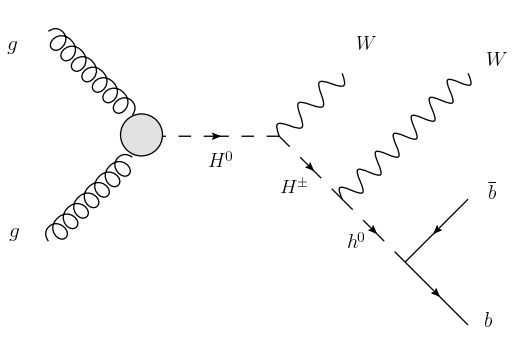

(a)

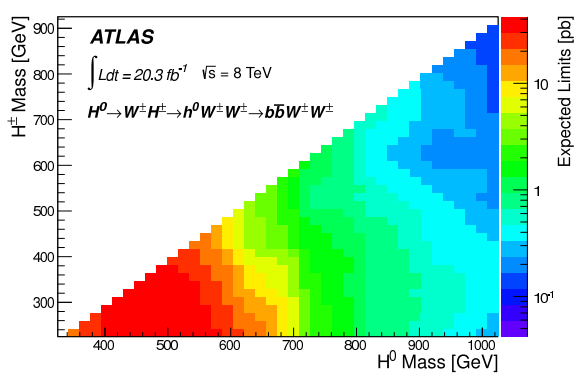

(b)

Figure 4. Cascade decay $H^{0} \rightarrow W H^{ \pm} \rightarrow h^{0} W W \rightarrow b \bar{b} W W$ [9]. (a) Feynman diagram; (b) The 95\% CL exclusion limit on the cross section times branching ratio as a function of Higgs boson masses.

\section{Neutral Higgs boson searches}

\subsection{Searches for invisible $H^{0}$ decays using associated $Z H$-production [10]}

In the Standard Model, production cross section of $Z h$ with subsequent decay of $h$ to $4 v$ is only 0.4 $\mathrm{fb}$ at $8 \mathrm{TeV}$, assuming $m_{h}=125 \mathrm{GeV}$. However, one can look at possible enhancements due to some BSM processes. An example of such a process is shown in Fig. 5a, where a neutral Higgs boson decays to a pair of neutralinos. The search mass range is $110-400 \mathrm{GeV}$. The signature is a pair of isolated same-flavour high- $p_{T}$ leptons with the invariant mass equal to the $Z$-boson mass and large $E_{T}^{m i s s}$. Backgrounds include $W W \rightarrow \ell v \ell v$ and $Z Z \rightarrow \ell \ell v v$ continuum production, $W Z$, single and pair top production and, in addition, the $h^{0}$ decays to $W W$ and $Z Z$ with the same final state. No significant excess over the background is observed over the whole mass range. $E_{T}^{\text {miss }}$-distributions are used to extract the limits on $\sigma_{Z H} \times B R(H \rightarrow$ invisible $)$. The limits as a function of Higgs boson mass are given in Fig. 5b. It was concluded that the $B R(H \rightarrow$ invisible $)$ is less than $75 \%$ at $95 \% \mathrm{CL}$.

\subsection{Search for $H \rightarrow W W \rightarrow \ell v \ell v$ at high mass [11]}

The search for $H \rightarrow W W \rightarrow \ell v \ell v$ in the high-mass region $260-1000 \mathrm{GeV}$ is performed. The predicted $\sigma_{H} \times B R(H \rightarrow W W \rightarrow \ell v \ell v)$ at $8 \mathrm{TeV}$ decreases from $300 \mathrm{fb}$ at $m_{H}=260 \mathrm{GeV}$ to $4 \mathrm{fb}$ at $m_{H}=1000$ $\mathrm{GeV}$. The selection criteria were specially optimized to high-mass searches and a cut-based analysis is performed. Only $H \rightarrow W W \rightarrow e v \mu \nu$ channel is considered. Two extreme Higgs boson lineshapes are taken: the SM-like, i.e. from the Complex Pole Scheme (CPS), and zero width, i.e. from the Narrow Width Approximation (NWA). Corresponding exclusion plots are shown in Fig. 6a and 6b, respectively. A SM-like Higgs boson with $m_{H} \leq 642 \mathrm{GeV}$ is excluded at $95 \% \mathrm{CL}$; a bit higher value is obtained for the NWA case. 


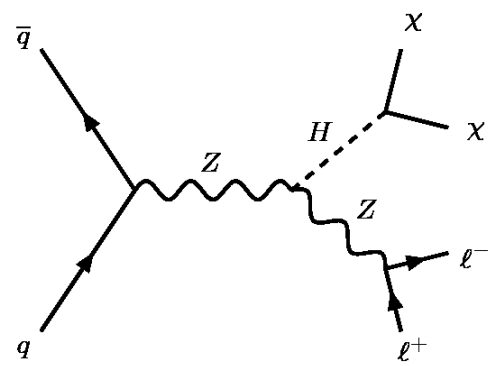

(a)

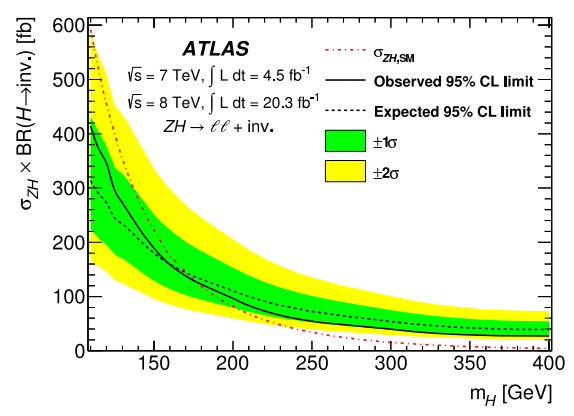

(b)

Figure 5. Searches for invisible $H^{0}$ decays using associated $Z H$-production [10]. (a) The Feynman diagram; (b) The $95 \%$ CL exclusion limit on the $\sigma_{Z H} \times B R(H \rightarrow$ invisible $)$ as a function of $m_{H}$.

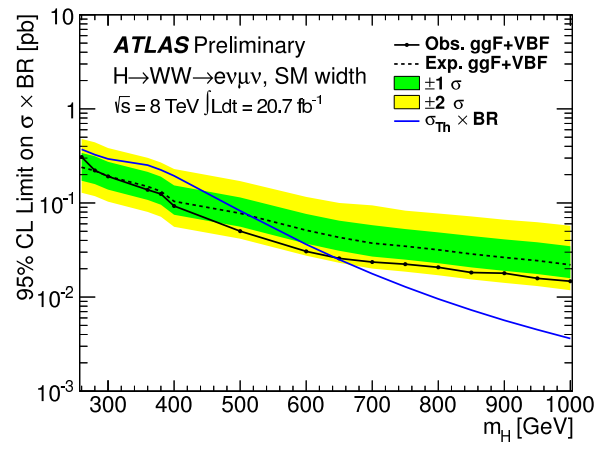

(a)

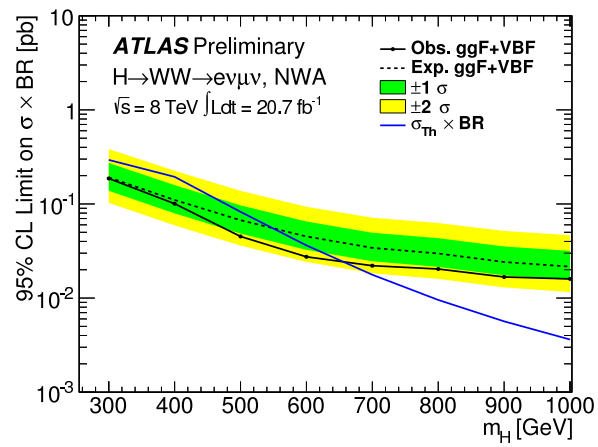

(b)

Figure 6. The 95\% CL upper limits on $\sigma_{H} \times B R(H \rightarrow W W \rightarrow e v \mu v)$ at $8 \mathrm{TeV}$ for a Higgs boson with (a) a SMlike lineshape and (b) a narrow lineshape (NWA). The green and yellow bands show the $1 \sigma$ and $2 \sigma$ uncertainties on the expected limit. The expected cross section times branching ratio for the production of a SM Higgs boson is shown as a blue line.

\subsection{Search for $H \rightarrow Z Z^{\star} \rightarrow 4 \ell$ at high mass [12]}

A search for $H \rightarrow Z Z^{\star} \rightarrow 4 \ell$ in the high-mass region $200-900 \mathrm{GeV}$ is performed. One would expect a low signal statistics because for the SM-case $\sigma_{H} \times B R(H \rightarrow 4 \ell)$ reduces from $5.5 \mathrm{fb}$ at $m_{H}=300$ $\mathrm{GeV}$ to less than $1 \mathrm{fb}$ at $m_{H}=600 \mathrm{GeV}$ at the CM energy of $8 \mathrm{TeV}$. The signature is two pairs of isolated same-flavour high- $p_{T}$ leptons; for both lepton pairs, the invariant mass should be compatible with the $Z$ boson mass. To increase the signal-to-background ratio and to look at different Higgs boson production mechanisms, the selected events are subdivided into three categories with the signatures close to gluon fusion $(\mathrm{ggF})$, vector boson fusion $(\mathrm{VBF})$ and associated $V H$-production. Irreducible backgrounds come from $q \bar{q}+g g \rightarrow Z Z^{\star}$ processes. Smaller reducible backgrounds are due to $Z+j e t s$ - 
and $t \bar{t}$-production. The four-lepton invariant mass distribution is shown in Fig. 7a. A SM-like Higgs boson with $m_{H} \leq 650 \mathrm{GeV}$ is excluded at $95 \% \mathrm{CL}$ in the ggF production mode (Fig. $7 \mathrm{~b}$ ), while in the VBF production mode the exclusion region is much narrower (Fig. 7c).

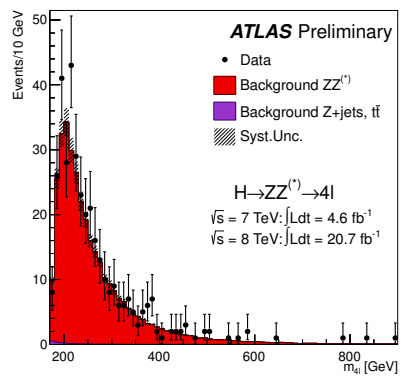

(a)

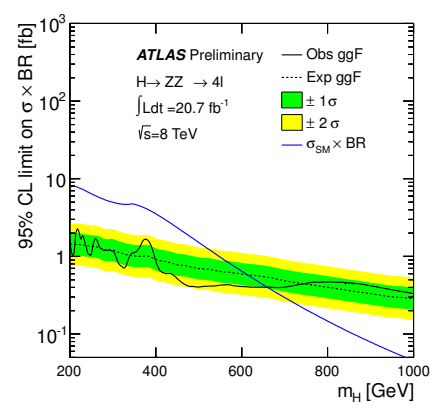

(b)

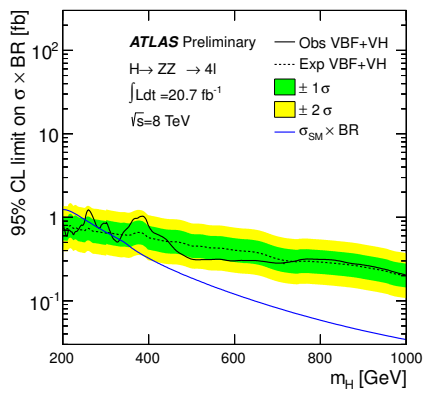

(c)

Figure 7. Search for $H \rightarrow Z Z^{\star} \rightarrow 4 \ell$ at high mass [12] (a) The four-lepton invariant mass distribution; (b), (c) The $95 \%$ CL exclusion limits on $\sigma_{H} \times B R(H \rightarrow 4 \ell)$ assuming ggF (VBF) production mode, respectively.

\subsection{Search for $H \rightarrow \gamma \gamma$ at high mass [13]}

A search for $H \rightarrow \gamma \gamma$ in the high-mass region $65-600 \mathrm{GeV}$ is made. The signature is two isolated high- $p_{T}$ photons having invariant mass compatible with the $m_{H}$. The irreducible background comes from the continuum production of photon pairs via $q \bar{q}+g g \rightarrow \gamma \gamma$ mechanisms. These processes have about a hundred times larger rates than expected for the signal. Reducible backgrounds include mostly $\gamma-j e t$, and jet - jet production, with one or two jets misidentified as photons in the detector, as well as the Drell-Yan production. There are two separate search regions: $65 \mathrm{GeV} \leq M(\gamma \gamma) \leq 110 \mathrm{GeV}$ (low-mass) and $110 \mathrm{GeV} \leq M(\gamma \gamma) \leq 600 \mathrm{GeV}$ (high-mass). To increase the discovery potential, the events in the low-mass region are further subdivided into three independent categories having different photon conversion conditions. The di-photon invariant-mass distribution is shown in Fig. 8a. No big deviation from the SM is seen. Fig. 8b shows the $95 \%$ CL exclusion limit on the fiducial cross section times $B R(H \rightarrow \gamma \gamma)$. One can see a few $2 \sigma$-fluctuations.

\subsection{MSSM $H / h / A \rightarrow \tau \tau$ searches [14]}

The main mechanisms of $H / h / A$-production are the $\mathrm{ggF}$ and the associated $b$-production. The relative fraction of these mechanisms depends on the Higgs boson mass. The search mass ranges are 90$200 \mathrm{GeV}$ and $200-1000 \mathrm{GeV}$. The signal signature is two reconstructed $\tau$ leptons with the invariant mass compatible with $m_{\phi}{ }^{3}$. Depending on the $\tau$-pair decays, the final state has two different leptons $(e \mu)$, lepton and hadronic jet or two hadronic jets. The background includes $Z \rightarrow \tau \tau-, Z \rightarrow \ell \ell-$, top-, di-boson- and fake lepton production. The analysis is similar to the one presented for the SM $h \rightarrow \tau \tau$ searches [15], but optimized for different production mechanisms and Higgs boson masses. No significant excess of events above the background is observed. The 95\% CL exclusion limits on

\footnotetext{
${ }^{3} \phi$ stands for the $H / h / A$ throuhgout the note.
} 


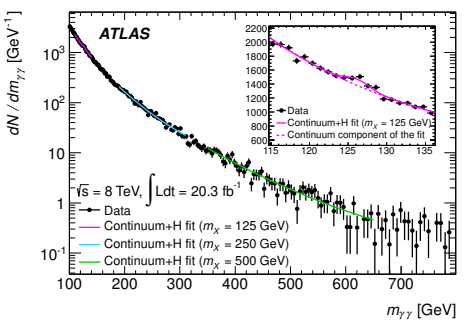

(a)

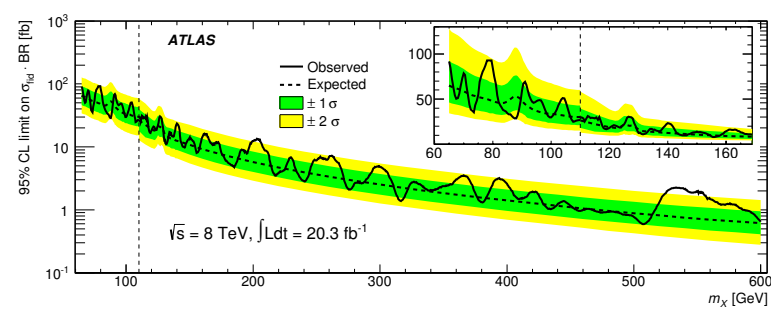

(b)

Figure 8. Searches for $H \rightarrow \gamma \gamma$ at high mass [13] (a) The di-photon invariant mass distribution; (b) The 95\% CL exclusion limits on $\sigma_{H} \times B R(H \rightarrow \gamma \gamma)$.

$\tan \beta$ as a function of $m_{A}$ are interpreted in terms of the MSSM model with a specific set of parameters (so-called $m_{h}^{\max }$ scenario and $M_{S U S Y}=1 \mathrm{TeV}$ ), see Fig. 9a. The best $\tan \beta$ constraints for combined searches are $\tan \beta \geq 5.4$ for $m_{A}=140 \mathrm{GeV}$ and $\tan \beta \geq 36$ for $m_{A}=800 \mathrm{GeV}$. $m_{A} \leq 160 \mathrm{GeV}$ is excluded for all $\tan \beta$ values and $\tan \beta \geq 10$ is excluded for all $m_{A}$ values provided the $h$ at $125 \mathrm{GeV}$ is associated with the given MSSM scenario. Fig. 9b shows that the excluded range of $\sigma_{\phi} \times B R(\phi \rightarrow \tau \tau)$ extends from $>29 \mathrm{pb}$ at $m_{\phi}=90 \mathrm{GeV}$ to $>7.4 \mathrm{fb}$ at $m_{\phi}=1000 \mathrm{GeV}$ for the ggF production mechanism. The same numbers for the b-associated production are $>6.4 \mathrm{pb}$ at $m_{\phi}=90 \mathrm{GeV}$ and $>7.2 \mathrm{fb}$ at $m_{\phi}=1000$ $\mathrm{GeV}$ (Fig. 9c).

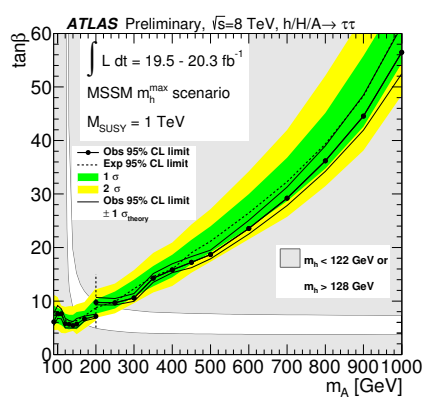

(a)

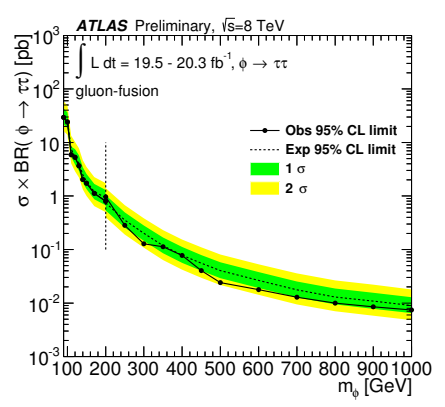

(b)

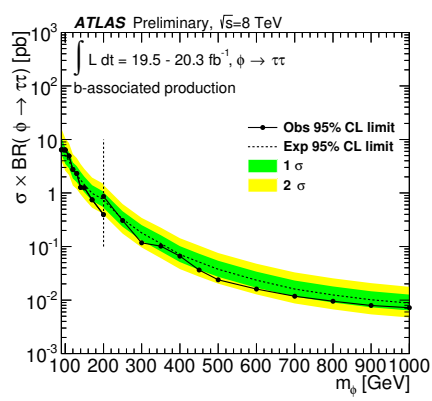

(c)

Figure 9. MSSM $H / h / A \rightarrow \tau \tau$ exclusion [14]. (a) The 95\% CL limits on $\tan \beta$ as a function of $m_{A}$ mass interpreted in terms of the MSSM model with specific set of parameters [8]. (b-c) The limits on $\sigma_{\phi} \times B R(\phi \rightarrow \tau \tau)$ in the $\mathrm{ggF}$ and $\mathrm{b}$-associated production mechanisms.

\section{6 $X \rightarrow h h \rightarrow b \bar{b} \gamma \gamma$ search at low and high masses [16]}

The search mass range for the resonant and non-resonant $h h \rightarrow b \bar{b} \gamma \gamma$ production is $260-500 \mathrm{GeV}$. Note that the expected SM cross section of this process is too small to be observed; only 0.04 signal 
events are predicted in the full $8 \mathrm{TeV}$ dataset. However, in some extensions of the $\mathrm{SM}$ the cross section could be higher. The signature is two high- $p_{T}$ isolated photons and two energetic jets, with diphoton and di-jet invariant masses compatible with the $m_{h}=125 \mathrm{GeV}$. The backgrounds (continuum and single $h$ production) are not big due to good di-photon invariant mass resolution, yielding the total of 1.5 events. $2.4 \sigma$ excess is seen in the non-resonant searches. 5 events are observed. The $95 \% \mathrm{CL}$ upper limit on the $\sigma_{X} \times B R(X \rightarrow h h)$ is between $0.8 \mathrm{pb}$ and $3.5 \mathrm{pb}$; for non-resonant production it is set to $2.2 \mathrm{pb}$.

\section{Conclusion}

ATLAS performed searches for Beyond the Standard Model Higgs bosons in many decay channels, using the $7 \mathrm{TeV}$ and $8 \mathrm{TeV}$ datasets:

- $H^{ \pm}$bosons in $\tau v$ - [7] and $c \bar{s}$-channels [6], as well as in cascade $H$ decays [9];

- High mass $H^{0}$ decays to $W W \rightarrow \ell v \ell v$ [11], $Z Z \rightarrow 4 \ell$ [12] and $\gamma \gamma$ [13];

- $H^{0} \rightarrow$ invisible decay using associated $Z H$-production [10];

- $H / h / A$ decays to $\tau \tau[14]$;

- $H \rightarrow h h$-production with subsequent $h$ decays to $b \bar{b}$ and $\gamma \gamma$ [16].

No deviations from the Standard Model were found, and strict limits on production cross sections and branching ratios were set, thereby limiting the parameter space of related theoretical models. ATLAS continues the search for BSM Higgs bosons in the 7 and $8 \mathrm{TeV}$ data using new channels and improved analyses. It is expected to obtain much better results with the new data from future LHC runs at 13-14 $\mathrm{TeV}$.

The work is partially supported by MES of Russia, grant RFMEFI61014X0005.

\section{References}

[1] ATLAS Collaboration, Phys. Lett. B716, 1 (2012)

[2] CMS Collaboration, Phys. Lett. B716, 30 (2012)

[3] L. Evans and P. Bryant (editors), Journal of Instrumentation 3, S08001 (2008)

[4] K.A. Olive et al. (Particle Data Group), Chin. Phys. C38, 090001 (2014)

[5] ATLAS Collaboration, Journal of Instrumentation, 3, S08003 (2008)

[6] ATLAS Collaboration, Europ. Phys. J. C73, 2465 (2013)

[7] ATLAS Collaboration, ATLAS-CONF-2013-090

[8] S. Heinemeyer, W. Hollik, and G. Weiglein, JHEP 0006, 209 (2000); M. Carena, S. Heinemeyer, C. E. M. Wagner and G. Weiglein, Eur. Phys. J. C26, 601 (2003)

[9] ATLAS Collaboration, PR D89, 032002 (2014)

[10] ATLAS Collaboration, Phys. Rev. Lett. 112, 201802 (2014)

[11] ATLAS Collaboration, ATLAS-CONF-2013-067, https://cds.cern.ch/record/1562879

[12] ATLAS Collaboration, ATLAS-CONF-2013-013, https://cds.cern.ch/record/1523699

[13] ATLAS Collaboration, arxiv 1407.6583, accepted for publication in Phys. Rev. Lett.

[14] ATLAS Collaboration, ATLAS-CONF-2014-049, https://cds.cern.ch/record/1744694

[15] ATLAS Collaboration, ATLAS-CONF-2013-108, https://cds.cern.ch/record/1632191

[16] ATLAS Collaboration, arxiv 1406.5053. 